\title{
Lipid Metabolic Events Underlying the Formation of the Corneocyte Lipid Envelope
}

\author{
Philip W. Wertz \\ University of lowa, lowa City, IA, USA
}

\section{Keywords \\ Corneocyte lipid envelope - Acylglucosylceramide . \\ Acylceramide $\cdot \omega$-Hydroxyceramide $\cdot$ Stratum corneum . \\ Barrier function}

\begin{abstract}
Cornified cells of the stratum corneum have a monolayer of an unusual lipid covalently attached to the outer surface. This is referred to as the corneocyte lipid envelope (CLE). It consists of a monolayer of $\omega$-hydroxyceramides covalently attached to the outer surface of the cornified envelope. The CLE is essential for proper barrier function of the skin and is derived from linoleate-rich acylglucosylceramides synthesized in the viable epidermis. Biosynthesis of acylglucosylceramide and its conversion to the cornified envelope is complex. Acylglucosylceramide in the bounding membrane of the lamellar granule is the precursor of the CLE. The acylglucosylceramide in the limiting membrane of the lamellar granule may be oriented with the glucosyl moiety on the inside. Conversion of the acylglucosylceramide to the CLE requires removal of the glucose by action of a glucocerebrosidase. The ester-linked fatty acid may be removed by an as yet unidentified esterase, and the resulting
\end{abstract}

$\omega$-hydroxyceramide may become ester linked to the outer surface of the cornified envelope through action of transglutaminase 1. Prior to removal of ester-linked fatty acids, linoleate is oxidized to an epoxy alcohol through action of 2 lipoxygenases. This can be further oxidized to an epoxyenone, which can spontaneously attach to the cornified envelope through Schiff's base formation. Mutations of genes coding for enzymes involved in biosynthesis of the CLE result in ichthyosis, often accompanied by neurologic dysfunction. The CLE is recognized as essential for barrier function of skin, but many questions about details of this essentiality remain. What are the relative roles of the 2 mechanisms of lipid attachment? What is the orientation of acylglucosylceramide in the bounding membrane of lamellar granules? Some evidence supports a role for CLE as a scaffold upon which intercellular lamellae unfold, but other evidence does not support this role. There is also controversial evidence for a role in stratum corneum cohesion. Evidence is presented to suggest that covalently bound $\omega$-hydroxyceramides serve as a reservoir for free sphingosine that can serve in communicating with the viable epidermis and act as a potent broad-acting antimicrobial at the skin surface. Many questions remain.

(c) 2021 S. Karger AG, Basel

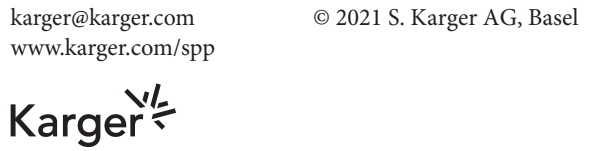

Philip W. Wertz, Professor Emeritus

University of Iowa

1412 Laurel Street

Iowa City, IA 52240 (USA)

philip-wertz@uiowa.edu 


\section{Introduction}

In the mid to late 1970s, a series of publications established that glucosylceramides accumulate with increasing differentiation in the epidermis and are deglycosylated to produce ceramides at the end of the differentiation process [1-3]. These studies established that the stratum corneum lipids consisted mainly of ceramides, cholesterol, and free fatty acids. They also identified that the sphingolipids from the pig and human epidermis were very similar. They contained sphingosine, dihydrosphingosine, and phytosphingosine bases and both normal and $\alpha$-hydroxyacids. The major glucosylceramide comprised about half of the total glucosylceramides and contained sphingosine and dihydrosphingosine bases amide linked to an unusual very long hydroxyacid with ester-linked linoleic acid [4]. It was later shown that the unusual fatty acid in this acylglucosylceramide (AGC) is mainly a series of 30-through 34-carbon $\omega$-hydroxyacids, and the linoleate is esterified to the $\omega$-hydroxyl group [5-7].

In 1983, the composition and detailed structures of the porcine epidermal glucosylceramides and ceramides were determined [6-8]. The ceramides included a linole- ate-rich acylceramide (AC), shown in Figure 1 (bottom), analogous to the unusual AGC, shown in Figure 1 (center) $[7,9,10]$. However, while the unusual linoleate-rich AGCs comprised $56 \%$ of the total glucosylceramide mass and, allowing for sphingomyelin, $41 \%$ of the total sphingolipid in the viable portion of the epidermis, the analogous AC made up only $7.7 \%$ of the ceramide mass in the stratum corneum. This raised the question of what happened to the missing $\omega$-hydroxyacylsphingosine and associated linoleic acid? It was subsequently found that the $\omega$-hydroxyceramide portion of the AGC becomes ester linked to the outer surface of the cornified envelope, shown in Figure 1 (top), and it was estimated that the amount of covalently bound $\omega$-hydroxyceramide was sufficient to completely envelop the corneocyte [11]. This expectation was supported by transmission electron microscopy of the thoroughly extracted stratum corneum [12]. An example is presented in Figure 2. This finding is supported by an earlier electron microscopy study of epidermal membranes that demonstrated that the lipid layer immediately adjacent to the corneocytes was distinctly different from other epidermal membranes in that it did not appear to contain cholesterol as judged by a filipin probe [13].

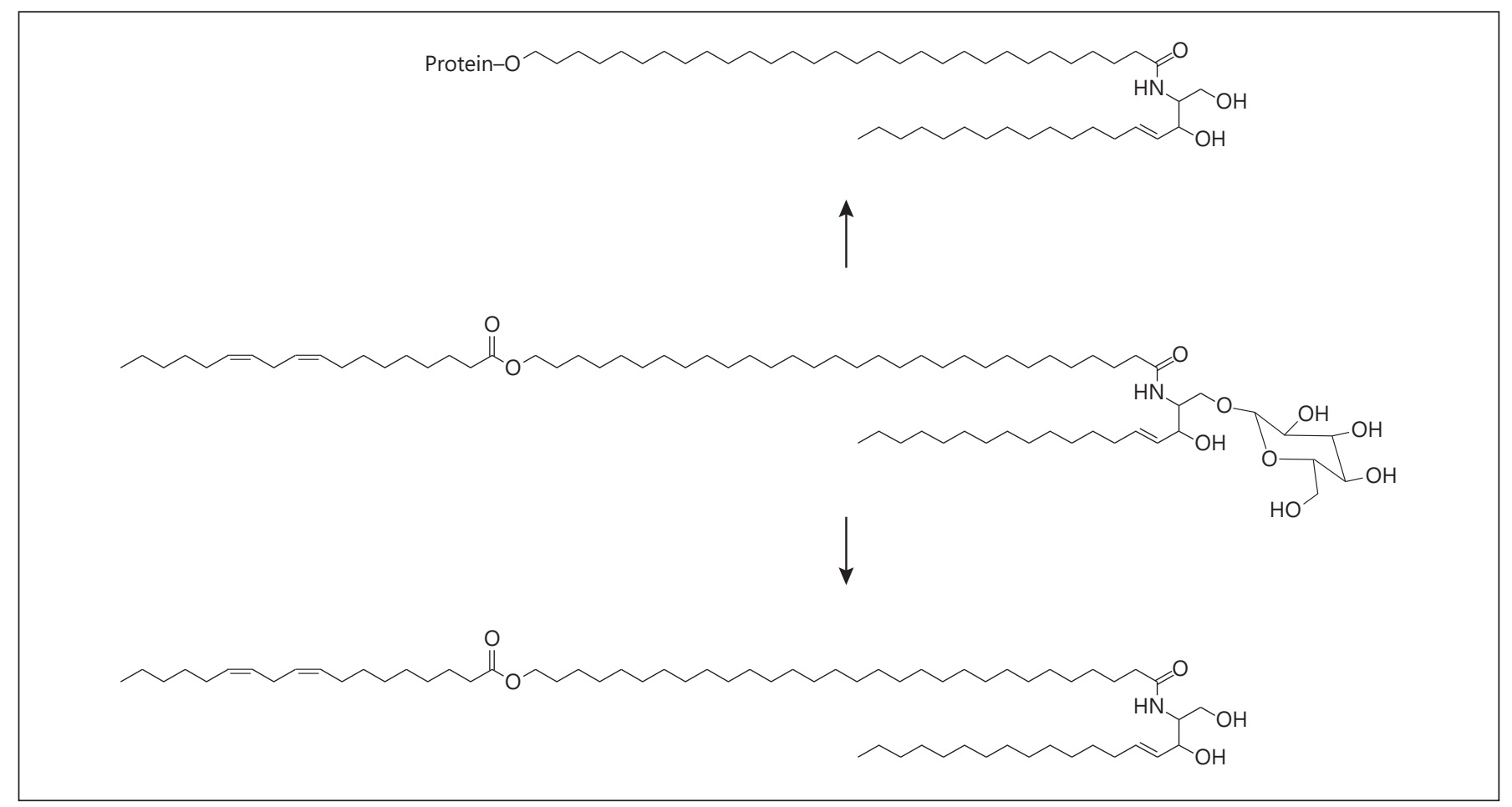

Fig. 1. AGC (center) is the precursor of the CLE (top) and the linoleate-rich AC (bottom). Reproduced from Wertz [54]. AGC, acylglucosylceramide; CLE, corneocyte lipid envelope; AC, acylceramide. 


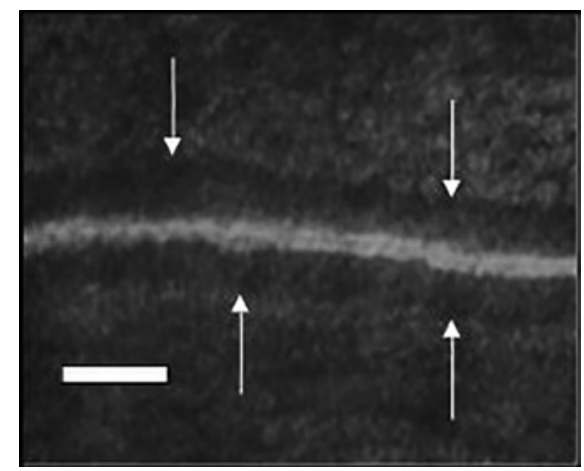

Fig. 2. After extensive extraction with chloroform:methanol mixtures, the transmission electron micrograph reveals that the free intercellular lipids have been removed and the intercellular spaces have collapsed. Arrows indicate the cornified envelopes of 2 adjacent cells. Lucent bands at the outer surfaces of the 2 cornified envelopes represent the CLEs. Bar, $50 \mathrm{~nm}$.

\section{Biosynthesis of $\boldsymbol{\omega}$-Hydroxyacids (Intracellular)}

In recent years, most of the steps in the biosynthesis of AGC have been elucidated. A significant part of this is the biosynthesis of long-chain $\omega$-hydroxyacids. Acetate from the circulation is the main source of carbon for synthesis of the very long fatty acids found in AGC as well as other lipids in the epidermis [14]. Acetate is converted to acetyl-CoA through the action of acetyl coenzyme A synthetase at the expense of 1 ATP with the release of pyrophosphate [15]. The biotin-dependent acetyl-CoA carboxylase converts acetyl-CoA into malonyl-CoA [16]. This reaction involves 2 consecutive steps $[16,17]$. The first step is the ATP-dependent formation of carboxybiotin. Then, the carboxyl group is transferred to acetyl-CoA to form malonyl-CoA. This is the rate-limiting step in fatty acid biosynthesis and is inhibited by phosphorylation and may be activated by diminished cholesterol [18]. Citrate is an allostearic activator of acetyl-CoA carboxylase. Acetyl-CoA and malonyl-CoA are the substrates for the multifunctional homodimeric cytosolic fatty acid synthase complex [19]. The mammalian fatty acid synthase includes acyl carrier protein (ACP), acetyl coenzyme A-ACP transacylase (acetyl transacylase) and malonyl coenzyme A-ACP transacylase (malonyl transacylase) activities, 3-ketoacyl-ACP synthase, 3-ketoacyl-ACP reductase, 3-hydroxyacyl-ACP dehydrase, enoyl-CoA reductase, and thioesterase. The reductase-catalyzed steps require NADPH. This cycle of condensation, reduction, dehydration, and reduction is repeated until palmitoyl-
ACP is obtained. Specificity is determined by thioesterase, which selectively releases palmitic acid [20]. The overall synthesis of palmitic acid is represented as

$$
\begin{aligned}
& \text { Acetyl-CoA + } 7 \text { malonyl-CoA + } 14 \mathrm{NADPH}+ \\
& 14 \mathrm{H}^{+} \rightarrow \mathrm{CH}_{3}\left(\mathrm{CH}_{2}\right)_{12} \mathrm{CO}_{2} \mathrm{H}+14 \mathrm{NADP}^{+} 7 \mathrm{CO}_{2}
\end{aligned}
$$

Palmitic acid can be converted to palmitoyl-CoA through the action of palmitoyl-CoA synthetase [21]. This reaction requires 1 ATP. Palmitoyl-CoA can then be converted to longer fatty acyl-CoAs through the action of fatty acid elongases located in the endoplasmic reticulum. There are 7 fatty acid elongases in mammals [22]. The mechanism of chain extension is similar to that involved in synthesis of palmitate by fatty acid synthase. It differs in that the substrates for each 2-carbon extension are fatty acyl-CoA and malonyl-CoA rather than the ACP thioesters. Each 2-carbon extension involves condensation, NADPH-dependent reduction, dehydration, and another NADPH-requiring reduction.

Each of the 7 elongases has characteristic substrate specificity [23]. Fatty acid elongases 2 and 5 are exclusively involved in elongation of polyunsaturated fatty acids, while fatty acid ELOVs 1, 3, 4, 6, and 7 are involved in chain extension of saturated and monounsaturated fatty acids [24]. In considering the biosynthesis of the C30:0 $\omega$-hydroxyacid that is the most abundant amide-linked fatty acid in the AGC, we start with palmitoyl-CoA [25]. ELOV6 primarily mediates the conversion of palmitoylCoA (C16:0) to stearoyl-CoA (C18:0). A combination of ELOV 3 and ELOV1 catalyzes conversion of stearoyl-CoA (C18:0) to arachidonyl-CoA (C20:0). ELOV1 catalyzes chain extension to produce certoyl-CoA (C26:0). Further elongation steps to produce $\mathrm{C} 30: 0$ and longer chains are catalyzed exclusively by ELOV4 [26]. There is actually some controversy regarding conversion of $\mathrm{C} 24: 0$ to $\mathrm{C} 26: 0$. According to several sources, ELOV1 can convert C20:0 to C24:0, but not further [22, 27]. ELOV4 can accomplish chain extension from C26:0, but not from C24:0 [26]. However, according to a different source, ELOV1 can extend C20:0 through C28:0 [24]. The roles of ELOVs 1, 3, 4 , and 6 are summarized in Figure 3.

The saturated $\omega$-hydroxyacids in the AGC from the porcine epidermis range from 20 through 34 carbons in length, but the 30 -carbon entity is by far the dominant species [6]. Monoenoic and dienoic species represent 35 and $7 \%$, respectively, of the $\omega$-hydroxyacids in this lipid class. The dominant monoene is $\mathrm{C} 32: 1$, and the major diene is C34:2. The double bonds in these fatty acids would be introduced by a fatty acid desaturase [28]. These de- 


\begin{tabular}{|c|c|}
\hline $\begin{array}{c}\text { Acetyl-CoA } \rightarrow \text { FAS } \leftarrow \text { Malonyl-CoA } \\
\downarrow \\
\text { C16:0 } \\
\downarrow \text { palmitoyl-CoA synthase } \\
\text { C16:0-COA } \\
\downarrow \text { ELOV6 } \\
\text { C18:0-COA } \\
\downarrow \text { ELOV3 } \\
\text { C20:0-CoA } \\
\downarrow \text { ELOV } 1 \\
\text { C26:0-COA } \\
\downarrow \text { ELOV4 } \\
\text { C30:0-CoA } \\
\downarrow \text { fatty acyl-CoA thioesterase? } \\
\text { C30:0 } \\
\downarrow \text { P450 } \\
\omega-O H-C 30: 0 \\
\downarrow \text { FATP4 } \\
\omega-O H-C 30: 0-C o A \\
\downarrow \text { ceramide synthase } 3 \\
\omega-O H-C E R \\
\downarrow \text { PNPLA } 1 \\
\text { Acylceramide } \\
\downarrow \text { ceramide glucosyltransferase } \\
\text { AGC }\end{array}$ & $\begin{array}{l}{[35,36]} \\
{[37-39]} \\
{[43]} \\
{[46]} \\
{[45]}\end{array}$ \\
\hline
\end{tabular}

Fig. 3. Metabolic pathway for the biosynthesis of AGC with saturated or monoenoic $\omega$-hydroxyacids. AGC containing dienoic $\omega$-hydroxyacids may be synthesized by the same pathway, depending upon the locations of the double bonds.

saturases introduce cis double bonds at specific positions in the fatty acyl-CoA chain in a reaction that requires molecular oxygen and NADPH. Although the locations of the double bonds in the monounsaturated and diunsaturated epidermal $\omega$-hydroxyacids have not been determined, the only fatty acyl-CoA desaturase known to be expressed in the epidermis is a $\Delta 9$ desaturase $[29,30]$. This enzyme has the greatest substrate preferences for C18:0-CoA and C16:0-CoA [31]. This would suggest that the monoenoic $\omega$-hydroxyacyl-CoAs have the double bond in the $\omega-9$ or $\omega-7$ position, or a mixture of these 2 positions. Chain extension of the monoenoic fatty acids is analogous to that of the saturates, as shown in Figure 3. Although the $\Delta 9$ desaturase is often referred to as stearylCoA desaturase, it can act on fatty acyl-CoAs with 12through 19-carbon saturated fatty acyl chains $[28,32]$. Evidence that $\Delta 9$ desaturase may introduce a second double bond comes from an unusual source: the major components of horse sebum are giant-ring lactones [33]. When hydrolyzed, these lactones yield very long-chain $\omega$-hydroxyacids, some of which are dienoic. The double bonds in 34-, 36-, and 38-carbon dienoic $\omega$-hydroxyacids derived from horse sebum have been shown to be consistent with the action of a $\Delta 9$ desaturase acting at the $\mathrm{C} 18$ stage of elongation followed by a second desaturation at the C24, C26, C28, C30, or C32 stage [34].

It has been reported that in the ELOV4 ${ }^{-/-}$mouse, the long-chain fatty acids C26:0, C26:1, and C26:2 are all elevated relative to the levels in the wild-type mouse epidermis [35]. This implies that ELOV4 is capable of elongating saturated, monounsaturated, and dienoic C26 fatty acids to ultimately produce the long-chain $\omega$-hydroxyacids found in the AGCs. Further work is needed to clarify details of the biosynthetic pathway leading to the long-chain dienoic fatty acids that are the precursors of some of the $\omega$-hydroxyacids found in epidermal sphingolipids.

Once the appropriate length of fatty acyl-CoA has been attained, the fatty acid is released by a thioesterase, and the terminal carbon is hydroxylated by a P450 coded by the CYP4F22 gene [36, 37]. This cytochrome P450 does not use the acyl-CoA as a substrate, necessitating release of the free acid. The free $\omega$-hydroxyacid is then converted back to a CoA thioester. The $\omega$-hydroxylated fatty acid-CoA serves as a substrate for a ceramide synthase. The enzyme that catalyzed the reformation of the CoA thioester is fatty acid transport protein 4 (FATP4), which also has acyl-CoA synthetase activity [38-40]. The reaction requires ATP.

\section{Biosynthesis of the Long-Chain Bases and Assembly of AGC (Intracellular)}

The rate-limiting step in the synthesis of all sphingolipids is catalyzed by serine palmitoyl transferase [41]. This enzyme condenses serine with palmitoyl-CoA in an $\mathrm{NADPH}$-dependent reaction that results in 3-ketodihydrosphingosine. An NADPH-dependent 3-ketodihydrosphingosine reductase reduces the keto group to produce dihydrosphingosine. Dihydrosphingosines are rapidly incorporated into ceramides through the action of ceramide synthases [42]. There are 6 ceramide synthases that differ in their fatty acid chain-length selectivity [43]. In the case of the very long-chain $\omega$-hydroxyacylceramide, ceramide synthase 3 would condense the $\omega$-hydroxyacylCoA with sphinganine to produce an $\omega$-hydroxyceramide [44]. After formation of the dihydrosphingosine-containing ceramide, a trans-double bond can be introduced between carbons 4 and 5 of the base to produce a sphingosine-containing ceramide or it can be hydroxylated on the 4-carbon to produce phytosphingosine-containing ceramide. Sphingosine can be hydroxylated on the 6-carbon to produce 6-hydroxysphingosine [45]. The $\omega$-hydroxyceramides are next glycosylated by a ceramide 
glucosyltransferase located in the Golgi apparatus [46]. A glucosylceramide containing very long-chain $\omega$-hydroxyacids amide linked to a mixture of sphingosine and dihydrosphingosine bases represents about $1.5 \%$ of the total glucosylceramides in the viable portion of the porcine epidermis $[6,10]$. This is converted to the AGC through the action of a transacylase, PNPLA1, which transfers linoleate from a triglyceride to the $\omega$-hydroxyl group [47-49]. The $\alpha / \beta$-hydrolase domain-containing 5 (ABHD5) interacts with PNPLA1 and acts as an activating cofactor for PNPLA1 [50]. Although it has been convincingly demonstrated that PNPLA 1 can transfer linoleate from triglycerides to the $\omega$-hydroxyl group, there is some possibility to consider that a phosphoglyceride may be a linoleate source in vivo [51]. For one thing, triglycerides represent $<1 \%$ of the total lipid in the pig epidermis. One day after intradermal injection of uniformly ${ }^{14} \mathrm{C}$-labeled linoleic acid, triglycerides had a very high specific activity, but the phospholipids contained $6.7 \times$ more radioactivity. Radioactivity of the triglyceride fraction gradually declined to zero by 21 days after injection. Radioactivity in the phospholipids peaked at the third day after injection. Radioactivity in the AGC peaked at about 7 days, and radioactivity in the $\omega-\mathrm{O}-\mathrm{AC}$ peaked at about 14 days. This scenario suggests that the triglyceride fraction may be involved in the initial uptake of linoleate with rapid transfer to phosphoglycerides. This is presumably mediated by conversion of triglyceride to diglyceride and incorporation into phosphoglycerides. Subsequently linoleate may be transferred from phosphoglyceride to the AGC. The AC is subsequently produced by deglycosylation of AGC. Also, the genome of the malaria parasite, Plasmodium falciparum, codes for 4 PNPLAs, including PNPLA1 [52]. When PNPLA1 was knocked out of the $P$. falciparum genome, it was found that during the blood phase, when the parasite is inside erythrocytes, the cells infected with the KO parasite contained significantly more phosphatidylcholine and less lysophosphatidic acid. This suggests the possibility that phosphatidylcholine and phosphatidic acid may serve as substrates for PNPLA1 in this setting. The major steps in the biosynthesis of AGC with saturated $\omega$-hydroxyacids are summarized in Figure 3.

\section{AGC in Lamellar Granules}

AGC is associated with epidermal lamellar granules, which arise from the Golgi apparatus [53-55]. Epidermal lamellar granules, as shown in Figure 4, are round to

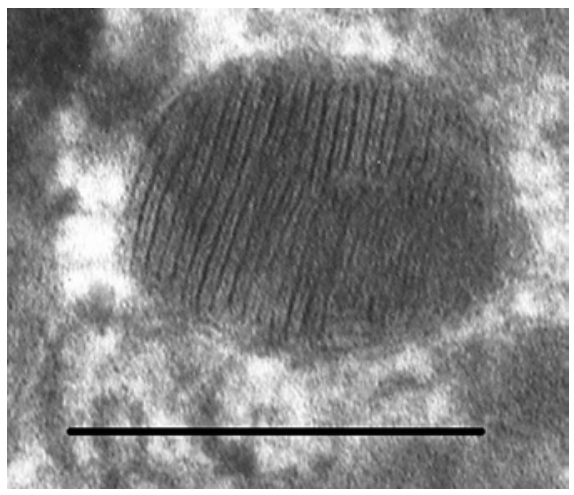

Fig. 4. A transmission electron micrograph of an epidermal lamellar granule. Scale bar, $200 \mathrm{~nm}$. Reproduced from Wertz [54].

ovoid in shape with a diameter of about $2 \mu \mathrm{m}$. They consist of a unit bounding membrane and contain stacks of lamellar lipid disks. Epidermal lamellar granule-enriched fractions have been isolated and partially characterized by several groups $[53,56,57]$. In addition to being lipid rich, the lamellar granules contain a number of hydrolytic enzymes $[53,57,58]$. At the boundary between the uppermost granular cells and the stratum corneum, the bounding membrane of the lamellar granule fuses into the cell plasma membrane, and the contents of the lamellar granule are extruded into the intercellular space. At this time, a glucocerebrosidase converts glucosylceramides into ceramides, and an acid sphingomyelinase likewise converts sphingomyelin into ceramides. Fatty acids are released from phosphoglycerides as the original plasma membrane is degraded. Effectively, the plasma membrane becomes replaced by an AGC-rich membrane derived from the bounding membrane of the lamellar granule.

AGC associated with the lamellar granules is the precursor of the covalently bound $\omega$-hydroxyceramides of the CLE. Although it seemed obvious that AGC from the bounding membrane of the lamellar granules would be the precursor of the covalently bound $\omega$-hydroxyceramide, this was not formally stated until 1992 [59]. More direct proof of this relationship came from electron microscopic studies of the stratum corneum from subjects with harlequin ichthyosis [60]. Harlequin ichthyosis results from a loss of function mutation of the lipid transporter gene $A B C A 12$ [61]. As a result of this mutation, lamellar granules are formed that lack internal lamellar lipid contents [62] These empty lamellar granules fuse into the plasma membrane and discharge their contents, presumably hydro- 


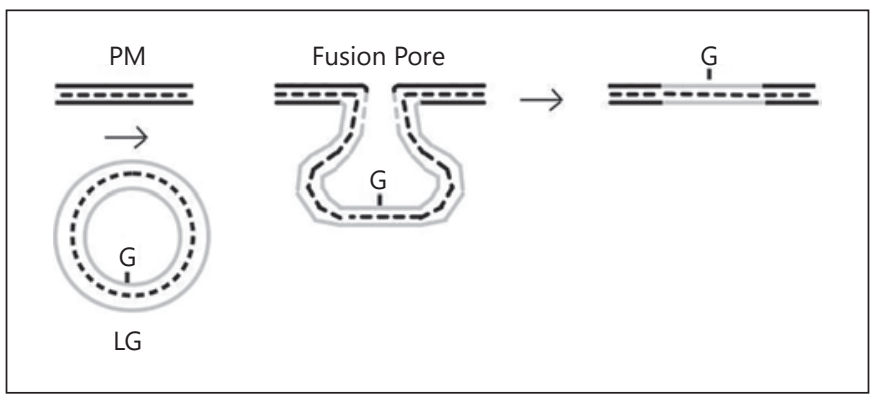

Fig. 5. Fusion of the bounding membrane of an LG with the residual PM proceeds by formation of a fusion pore (center) to integrate the bounding membrane of the LG into the PM (right). G indicates the location of the glucosyl moiety of AGC. LG, lamellar granule; PM, plasma membrane; AGC, acylglucosylceramide.

lytic enzymes, into the extracellular space, and the existence of a CLE was revealed by transmission electron microscopy [60].

AGC from the viable portion of the epidermis and the $\mathrm{AC}$ found in the stratum corneum have nearly identical $\omega$-hydroxyacids and ester-linked fatty acids, including 74-75\% ester-linked linoleate. If there were selectivity for linoleate-containing AGC for the bounding membrane of the lamellar granule, it would be expected that the linoleate content of the $\mathrm{AC}$ would be much lower. If linoleatecontaining AGC was selected for the bounding membrane of the lamellar granule, the ester-linked fatty acids of the AC would have been expected to be approximately $14.2 \%$ linoleate, $36.4 \%$ oleate, and $39.4 \%$ saturated fatty acids. Therefore, the AGC in the bounding membrane of the lamellar granule includes species with saturated and monoenoic ester-linked fatty acids in addition to linoleate. The $\omega$-hydroxyacids from the covalently bound hydroxyceramides range from 28 - through 34 -carbons in length $[11,63,64]$. Given the relative amounts of AGC in the viable epidermis and the amount of $\mathrm{AC}$ in the stratum corneum, it follows that about $80 \%$ of the AGC associated with the lamellar granule is in the bounding membrane, while about $20 \%$ is in the internal lamellae.

Consider the arrangement of AGC in the bounding membrane of the lamellar granule. It would be expected that the AGC in the bounding membrane of the lamellar granule would be oriented with the glucose at the outer surface. It is generally the case that plasma membranes and biological vesicles have glycolipids oriented in this manner. The very long extended $\omega$-hydroxyacids in the AGC present a mechanistic problem for membrane fusion. In addition, very long-chain fatty acids in the phosphoglycerides of the plasma membrane and la-

Lipid Metabolic Events in the Formation of CLE mellar granules also present a problem for membrane fusion because they preclude clearly defined inner and outer bilayer leaflets of the plasma membrane. The free fatty acids of the stratum corneum are derived from these phosphoglycerides, and about two-thirds of the free fatty acids from the stratum corneum contain 20 or more carbons with C22:0 and C24:0 as the most abundant species. These long-chain fatty acids in the phosphoglycerides extend beyond the midpoint of the bilayer to interdigitate with lipids from the opposing leaflet. The most widely accepted mechanism for fusion of biological membranes involves the inner leaflet of one membrane and the outer leaflet of the other membrane forming a hemifusion stalk leading ultimately to formation of a fusion pore [61]. This is illustrated in Figure 5. The fusion of membranes in vivo is facilitated by certain proteins including SNAPs and Rabs, which have been identified in fractions enriched in epidermal lamellar granules isolated from the human epidermis [57]. The long $\omega$-hydroxyacids of the AGCs and the long fatty acids in phosphoglycerides present a problem for membrane fusion because they preclude clearly defined inner and outer bilayer leaflets. Phosphoglyceride-rich domains containing the remaining C16 and C18 fatty acids must segregate within both the plasma membrane and the bounding membrane of the lamellar granule and come together to form the hemifusion stalk. Once the fusion pore has formed, the contents of the lamellar granule can be discharged through the pore, and the bounding membrane of the lamellar granule flows into the residual plasma membrane. An obvious problem with the arrangement with the AGC on the outer surface of the bounding membrane of the lamellar granule and the $\omega$-hydroxyl group at the inner surface of the bounding membrane is that after fusion these positions would be reversed. The glucosyl moiety would be facing the nascent corneocyte envelope instead of the just extruded $\beta$-glucocerebrosidase, and the $\omega$-hydroxyl group, still with ester-linked linoleate, would not be adjacent to the nascent corneocyte envelope and the transglutaminase.

However, there is some evidence indicating that the AGC may have the glucosyl moiety on the inner side of the bounding membrane as shown in Figure 5. Somewhat surprisingly, lamellar granules have been isolated from epidermis that has been frozen at $-20^{\circ} \mathrm{C}$ and then thawed [46]. Slow freezing usually disrupts cells and all internal organelles through the formation of ice crystals. The fact that lamellar granules are not disrupted by this process suggests that they have a very low free water content. This 
is also consistent with the fact that they contain various hydrolytic enzymes along with their substrates, but hydrolysis does not take place until these materials are expelled from the granules. If AGC in the bounding membrane is oriented with the glucosyl moiety on the inside, this could help in reducing water activity through hydrogen bonding. This orientation is further supported by the observation that TEMPO radicals failed to oxidize any of the AGC from isolated lamellar granules, while under the same conditions, liposomes containing glucosylceramide underwent extensive oxidation [65]. From the size of the liposomes, it was estimated that 60 percent of the glucosylceramide would be in the outer leaflet with 40 percent in the inner leaflet. The percent that was oxidized by TEMPO was 62 percent. This glucose-inside orientation, as illustrated in Figure 5, would potentially allow the linoleate to insert into, and thereby destabilize, the plasma membrane. This could occur at the outer edge of the nascent fusion pore and could be important for membrane fusion. There is ample evidence from studies of model membranes that the linoleate-containing sphingolipids can cause aggregation of lipid vesicles, presumably by insertion of the ester-linked fatty acid from an $\omega$-Oacylsphingolipid into an adjacent vesicle [66-68]. Such an insertion would also destabilize the outer leaflet of the bounding membrane thus facilitating fusion. As illustrated in Figure 5, if AGC in the bounding membrane of the lamellar granule is oriented with the glucose on the inside, then after fusion, the glucosyl moiety would be exposed to the just extruded glucocerebrosidase, and the $\omega$-hydroxyl group with esterified linoleate would face the nascent cornified envelope with associated transglutaminase 1 .

An alternative mechanism for formation of the stratum corneum barrier has been proposed [69]. In this membrane folding model, there is continuity between the membranes of the Golgi apparatus and the intercellular membranes of the stratum corneum. Lamellar granules do not exist as discrete vesicular organelles, but are part of a tubuloreticular network [70]. The formation of the lamellae in the intercellular spaces of the stratum corneum involves a cubic to lamellar phase lipid transition [71, 72]. Membrane fusion is not required in this model.

The membrane folding model was originally proposed on the basis of electron micrographs showing membrane transitions in a variety of biological specimens other than the epidermis [69]. The examples included skeletal muscle cells, plant chloroplasts UT-1 cells derived from Chinese ovary cells, and bronchioalveolar carcinoma. One transmission electron micrograph of ruthenium tetroxide-fixed epidermis showing lamellar granules was pres- ent, but was reinterpreted as representing a continuous cubic phase membrane system. Subsequent cryoelectron microscopic studies and EM simulation have provided some more direct support for this model [72]; however, image interpretation may be a limitation. Until cryotransmission microscopy becomes more widely applied to the study of the epidermis, the conflict between the conventional model of barrier formation and the membrane folding model will remain unresolved.

\section{Conversion of AGC to CLE (Extracellular)}

The next steps in formation of the CLE are catalyzed by $12 \mathrm{R}-\mathrm{LOX}$ and eLOX3 $[73,74] .12 \mathrm{R}$-Lipoxygenase and eLOX3 would likely be present in the plasma membrane because they are found at the periphery of epidermal cells and persist into the stratum corneum $[75,76]$. Their action on linoleate would apparently facilitate removal of this fatty acid, but the esterase or esterases that remove this and the other fatty acids have not been identified. The lipoxygenase, 12R-LOX, attacks the linoleate chain of the AGC to produce a hydroperoxide, and eLOX then acts as a hydroperoxide isomerase to produce a hydroxylated epoxide derivative, a hepoxilin. The epoxide moiety can then be opened through the action of an epoxide hydrolase to produce a trihydroxy derivative, or trioxilin [77]. These oxidized linoleate chains are thought to be substrates for an esterase leading to removal of the linoleate. The released triol may have a regulatory role in barrier homeostasis $[77,78]$. The production of linoleate-derived triols in the epidermis was first reported in 1985 [79]. It must be pointed out that $26 \%$ of the ester-linked fatty acids in AGCs are saturated or monounsaturated. Since the linoleate content of AGC from the viable portion of the epidermis and the AC from the stratum corneum both contain $74 \%$ linoleate, there would appear to be no selection for only linoleate-containing AGC molecules in making the CLE. The esterase or esterases remove the ester-linked fatty acid. The hepoxilins or trioxilins released from the sphingolipid may serve in regulation of some aspects of barrier formation [77]. It is not clear whether glucose is removed before or after attachment of the $\omega$-hydroxyceramide to the cornified envelope. It has been demonstrated that transglutaminase 1 can form ester linkages between an analog of the natural $\omega$-hydroxyceramide and involucrin [80]. This involved certain specific glutamine residues.

An alternative mechanism by which $\omega$-hydroxyceramide may become attached to the corni-
Skin Pharmacol Physiol 2021;34:38-50 DOI: $10.1159 / 000513261$
Wertz 
fied envelope is through SDR9C7 catalyzed oxidation of the hydroxyl group in the previously mentioned hepoxilin to a ketone, which can spontaneously react with free amino groups to attach the molecule to the cornified envelope [81]. This potential mechanism would only apply to attachment of $\omega$-hydroxyceramide derived from linoleate-containing AGC.

\section{Functions of the CLE}

It was initially proposed that the CLE provided an interface between the corneocyte and the intercellular lamellae which could be of importance for both the permeability barrier function and for coherence of the stratum corneum [11]. It was further suggested that the CLE could provide a barrier for passage of water into or out of the cornified cell [12]. This impermeability of the corneocytes would, of course, also be important for the tortuous diffusion pathway through the stratum corneum $[82,83]$. Soon after, it was speculated that the CLE could serve as a template upon which the extruded lamellar granule contents spread into broad multilamellar sheets [84] and that it could also explain the resistance of the stratum corneum to proteases [85].

Transmission electron micrographs of murine, porcine, and human stratum corneum using ruthenium tetroxide as a postfixative have revealed multilamellar lipid structures with 3-, 6-, and 9-lucent banded structures as the most abundant $[86,87]$. In each of these structures, the outermost lucent bands would represent the covalently bound $\omega$-hydroxyceramide. In each of these lamellar patterns, the lucent bands alternated broad-narrowbroad with an overall dimension of $13 \mathrm{~nm}$ [85]. This is the same dimension as the long periodicity for stratum corneum lipids obtained from X-ray diffraction studies $[87,88]$. A model was proposed for the 3-band structure in which sphingosines from covalently bound $\omega$-hydroxyceramide molecules everted to make the framework of a central bilayer with free lipids filling the remaining space [89]. It is conceivable that such an arrangement could contribute to cell-cell cohesion.

In 1989, an attempt was made to gain insights into the possible role of the CLE in cell-cell cohesion [90]. A detergent mixture was used that rapidly disaggregated the stratum corneum into individual corneocytes. The fact that the stratum corneum used in these experiments was isolated using trypsin makes it likely that proteolytic degradation of corneodesmosomes was part of the surfactant-induced disaggregation. Extraction of free lipids

Lipid Metabolic Events in the Formation of CLE

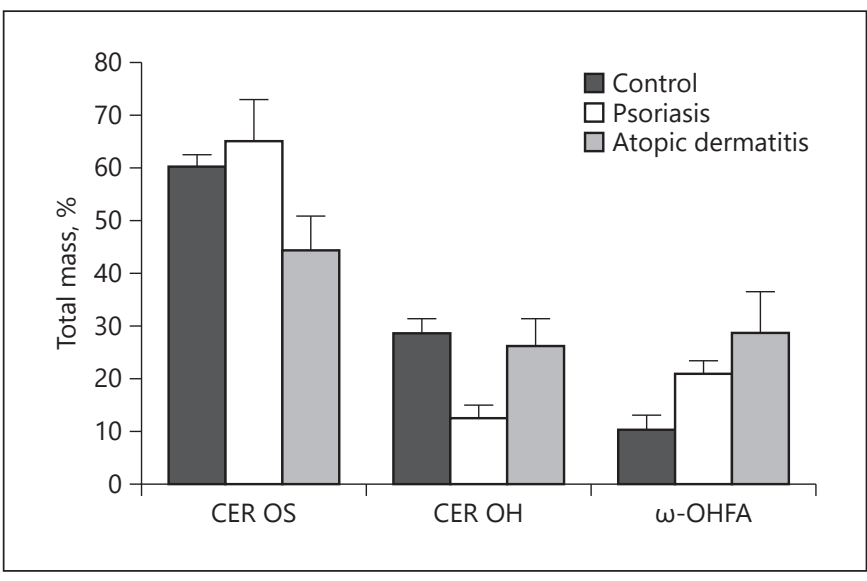

Fig. 6. Proportions of covalently bound ceramide OS, ceramide $\mathrm{OH}$, and $\omega$-hydroxyacid from the normal control stratum corneum and from psoriasis and atopic dermatitis.

prevented this disaggregation. Transmission electron microscopic examination of the detergent-treated extracted stratum corneum revealed regions, mainly near the ends of corneocytes, where lipid envelopes were closely apposed. It was suggested that when the free lipids were extracted, the trilaminar structures collapsed, and the covalently bound hydroxyceramides formed 2 closely apposed layers with sphingosine tails interdigitating to effectively hold the corneocytes together. Subsequently, stratum corneum cohesion was measured directly by cohesometry while progressively removing free lipids [91]. Progressive removal of lipids resulted in increasing cohesiveness which was attributed to the close apposition of the lipid envelopes as seen in the earlier study. It was suggested that under normal conditions, the envelopes would be kept apart by layers of free lipid lamellae. This does not take into consideration the previously noted interdigitated trilamellar structures and their possible collapse upon free lipid removal. The possible role of CLE contribution to cell cohesion remains unsettled.

Crumrine et al. [92] have presented evidence that the CLE is important for both proper formation of the intercellular lipid lamellae and the cornified envelope. In several human subjects with ARCI and an ALOX12 $2^{-/-}$mouse model, the CLE was attenuated or absent. Under these conditions, the intercellular lipid lamellae were not well organized, and the cornified envelope was thin. FATP4deficient mice also had a thinned cornified envelope and lacked a lipid envelope. Transgenic rescue of the CLE normalized the cornified envelope. However, in palatal and gingival stratum corneum, there is very little cova- 
Table 1. Mutations of human genes that compromise CLE formation [40, 47-50, 109-117]

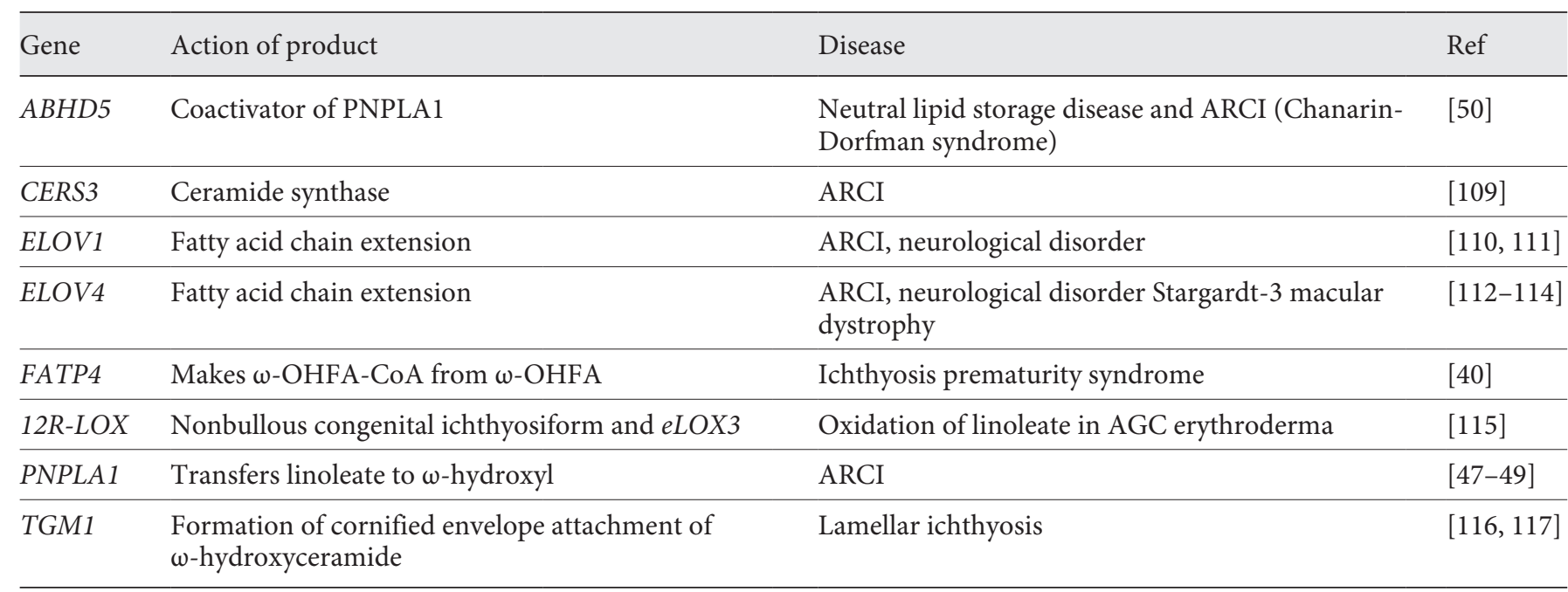

Mutations of genes involved in formation of the CLE cause ichthyosis and neuropathies. CLE, corneocyte lipid envelope; Ref, reference; ARCI, autosomal recessive congenital ichthyosis; $\omega$-OHFA, $\omega$-hydroxyacid; AGC, acylglucosylceramide.

lently bound $\omega$-hydroxyceramide, and there is no lipid envelope. Nevertheless, organized lamellae are seen sitting upon the cornified envelope [93]. The role, if any, of the CLE in conversion of the extruded lamellar granule contents into well-organized broad lamellae is still not settled.

The covalently bound $\omega$-hydroxyceramides are invariably accompanied by covalently bound $\omega$-hydroxyacids $[63,93]$. This is presumed to reflect the action of a ceramidase on the $\omega$-hydroxyceramide with the release of a long-chain base. Ceramidase activity has been demonstrated in the epidermis [94-96]. Free sphingosine, dihydrosphingosine, and 6-hydroxysphingosine have been detected in the epidermis, and the concentrations of free long-chain base were higher in the stratum corneum than in the viable portion of the epidermis, thus establishing a concentration gradient [97-99]. Free phytosphingosine has not been detected. This indicates that covalently bound CER[OS] and CER $[\mathrm{OH}]$ are potential ceramidase substrates, but covalently bound CER[OP] would not be a substrate. Long-chain bases are potent and broad-acting antimicrobials [100-103]. They are also potent inhibitors of protein kinase C [104]. It has been proposed that the sphingosine gradient may serve 2 functions - providing sphingosine as a potent antimicrobial to the skin surface and communication with the viable epidermis by means of sphingosine inhibiting protein kinase C [105]. Inhibition of protein kinase $\mathrm{C}$ would slow cell replication and differentiation. If one were to remove several layers of cells from the stratum corneum by tape stripping, it would reduce the concentration gradient. This would be expected to be followed by an increase in cell proliferation and differentiation until the injury was repaired. This is, in fact, what has been observed [106]. It would be expected that such a system would be responsive to conditions that impaired barrier function, thereby making more water available in the intercellular spaces of the stratum corneum. In accord with this expectation, in psoriasis and atopic dermatitis, the epidermis is somewhat hyperproliferative, and barrier function is impaired as judged by increased TEWL. In these conditions, hydrolysis of the covalently bound $\mathrm{OS}$ and $\mathrm{OH}$ is significantly elevated compared to normal healthy controls as shown in Figure 6 (Wertz, unpublished observations). Interestingly, most of the increase in $\omega$-hydroxyacid in atopic dermatitis comes from CER OS, while in psoriasis, it comes from CER OH. Furthermore, as essential fatty acid deficiency develops in young pigs, the TEWL increases [107]. As TEWL increases, the level of covalently bound ceramide OS decreases while the level of covalently bound $\omega$-hydroxyacid goes up proportionately [101]. The covalently bound $\omega$-hydroxyceramides may serve as a reservoir of long-chain bases to provide antimicrobial activity to the skin surface and to communicate needs for proliferation and differentiation to the viable epidermis. The possibility that free ceramides in the stratum corneum 
serve as sources of free long-chain bases seems unlikely in view of the fact that the proportions of the various ceramides remains unaltered from the top to the bottom of the stratum corneum [108].

\section{Mutations Affecting the Lipid Envelope Formations and Barrier Function}

The biosynthetic pathway leading to the formation of the CLE has been elucidated largely by identifying the enzymes coded by genes whose mutations are associated with ichthyoses. Crumrine et al. [92] have recently reviewed this area. A summary of gene mutations in humans that diminish cornified lipid envelope formation is given in Table 1.

\section{Unresolved Issues}

Although major steps in the biosynthesis of AGC and the CLE have been identified in recent years, there are still many details remaining unknown:

- What are the relative merits of transglutaminase-mediated versus epoxy-enone-mediated attachment of $\omega$-hydroxyceramide to the cornified envelope? Both mechanisms may contribute to CLE formation, but since the epoxy-enone-mediated attachment pertains only to linoleate-containing AGC, it cannot account for all covalent attachment. Available amino groups may also limit the epoxy-enone attachment.

- The conflicting views on chain extension from C24 to C26 need to be resolved.

- In the synthesis of the dienoic $\omega$-hydroxyacids, where are the 2 double bonds, and which ELOVs are involved in chain extension? If both double bonds are introduced to produce C26:2, then it appears that ELOV4 could complete chain extension. However, if the second double bond is introduced after the C26:1 stage, it is not clear which ELOV would complete extension.

- What is the identity of the fatty acyl-CoA thioesterase that releases very long fatty acids prior to $\omega$-hydroxylation?

- What is the in vivo substrate for PNPLA1? The current literature indicates linoleate is transferred from triglyceride to $\omega$-hydroxyceramide, but there is some evidence that the in vivo source of linoleate and other fatty acids may be phosphoglycerides.

- What is the orientation of AGC in the bounding membrane of lamellar granules? Evidence is presented to support an extended AGC with glucose on the inside of the bounding membrane; however, more work needs to be done to test this proposition.

- Are the removal of glucose and ester-linked fatty acids from the AGC sequential or simultaneous?

- What is the identity of the esterase or esterases involved in removal of the ester-linked fatty acids from the AGC or AC? Do different esterases act on oxidized linoleate versus monoenoic and saturated fatty acids?

- What is the role, if any, of the trioxilins derived from linoleate epoxy alcohol?

- Although the CLE is essential for barrier function of the skin, details of its function remain unsettled. What are its roles, if any, in organization of the intercellular lamellae of the intercellular spaces of the stratum corneum and in cohesion?

- More work needs to be done to examine release of sphingosine from covalently bound ceramides OS and $\mathrm{OH}$ under different conditions.

\section{Acknowledgements}

The author would like to acknowledge Nancy Wertz for editorial assistance in the preparation of this manuscript.

\section{Conflict of Interest Statement}

The author has no conflicts of interest regarding this work.

\section{Funding Sources}

No funding was provided for this work.

\section{References}

1 Gray GM, Yardley HJ. Lipid compositions of cells isolated from pig, human, and rat epidermis. J Lipid Res. 1975;16(6):434-40.

2 Gray GM, Yardley HJ. Different populations of pig epidermal cells: isolation and lipid composition. J Lipid Res. 1975;16(6):441-7.

3 Gray GM, White RJ. Glycosphingolipids and ceramides in human and pig epidermis. J Invest Dermatol. 1978;70(6):336-41.

4 Gray GM, White RJ, Major JR. 1-(3'-O-acyl)beta-Glucosyl-N-dihydroxypentatriacontdienoylsphingosine, a major component of pig and human epidermis. Biochim Piophys Acta. 1978;528:127-37.

5 Wertz PW, Downing DT. Glycolipids in the epidermis: structure and function in the water barrier. Science. 1982;217:1261-2. 
6 Wertz PW, Downing DT. Acylglucosylceramides of pig epidermis: structure determination. J Lipid Res. 1983;24(6):753-8.

7 Wertz PW, Downing DT. Ceramides of pig epidermis: structure determination. J Lipid Res. 1983;24(6):759-65.

8 Wertz PW, Downing DT. Glucosylceramides of pig epidermis: structure determination. J Lipid Res. 1983;24:1135-9.

9 Abraham W, Wertz PW, Downing DT. Linoleate-rich acylglucosylceramides of pig epidermis: structure determination by proton magnetic resonance. J Lipid Res. 1985;26(6): 761-6.

10 Bowser PA, Nugteren DH, White RJ, Houtsmuller UM, Prottey C. Identification, isolation and characterization of epidermal lipids containing linoleic acid. Biochim Biophys Acta. 1985;834(3):419-28.

11 Wertz PW, Downing DT. Covalently bound omega-hydroxyacylsphingosine in the stratum corneum. Biochim Biophys Acta. 1987; 917(1):108-11.

12 Swartzendruber DC, Wertz PW, Madison KC, Downing DT. Evidence that the corneocyte has a chemically bound lipid envelope. J Invest Dermatol. 1987;88(6):709-13.

13 Kitajima Y, Sekiya T, Mori S, Nozawa Y, Yaoita H. Freeze-fracture cytochemical study of membrane systems in human epidermis using filipin as a probe for cholesterol. J Invest Dermatol. 1985;84(2):149-53.

14 Hedberg CL, Wertz PW, Downing DT. The time course of lipid biosynthesis in pig epidermis. J Invest Dermatol. 1988;91(2):169-74.

15 Starai VJ, Escalante-Semerena JC. Acetyl-coenzyme A synthetase (AMP forming). Cell Mol Life Sci. 2004;61(16):2020-30.

16 Slabas AR, Brown A, Sinden BS, Swinhoe R, Simon JW, Ashton AR, et al. Pivotal reactions in fatty acid synthesis. Prog Lipid Res. 1994 33(1-2):39-46.

17 Brownsey RW, Zhande R, Boone AN. Isoforms of acetyl-CoA carboxylase: structures, regulatory properties and metabolic functions. Biochem Soc Trans. 1997;25(4):12328.

18 Smythe CD, Greenall M, Kealey T. The activity of HMG-CoA reductase and acetyl-CoA carboxylase in human apocrine sweat glands, sebaceous glands, and hair follicles is regulated by phosphorylation and by exogenous cholesterol. J Invest Dermatol. 1998;111(1):13948.

19 Jones SF, Infante JR. Molecular pathways: fatty acid synthase. Clin Cancer Res. 2015; 21(24):5434-8.

20 Chakravarty B, Gu Z, Chirala SS, Wakil SJ, Quiocho FA. Human fatty acid synthase: structure and substrate selectivity of the thioesterase domain. Proc Natl Acad Sci U S A. 2004;101(44): 15567-72.

21 Bar-Tana J, Rose G, Brandes R, Shapiro B. Palmitoyl-coenzyme A synthetase. Mechanism of reaction. Biochem J. 1973;131(2): 199-209.
22 Ohno Y, Suto S, Yamanaka M, Mizutani Y, Mitsutake S, Igarashi Y, et al. ELOVL1 production of C24 acyl-CoAs is linked to C24 sphingolipid synthesis. Proc Natl Acad Sci U S A. 2010;107(43):18439-44.

23 Jakobsson A, Westerberg R, Jacobsson A. Fatty acid elongases in mammals: their regulation and roles in metabolism. Prog Lipid Res. 2006;45(3):237-49.

24 Kihara A. Very long-chain fatty acids: elongation, physiology and related disorders. J Biochem. 2012;152(5):387-95.

25 Uchida Y. The role of fatty acid elongation in epidermal structure and function. Dermatoendocrinol. 2011;3(2):65-9.

26 Kitazawa H, Miyamoto Y, Shimamura K, Nagumo A, Tokita S. Development of a highdensity assay for long-chain fatty acyl-CoA elongases. Lipids. 2009;44(8):765-73.

27 Agbaga M-P, Brush RS, Mandal MNA, Henry $\mathrm{K}$, Elliott $\mathrm{MH}$, Anderson RE. Role of stargard-3 macular dystrophy protein (ELOV4) in the biosynthesis of very long chain fatty acids. Proc Natl Acad Sci U S A. 2008;105: 12843-8.

28 Nagao K, Murakami A, Umeda M. Structure and function of $\Delta 9$-fatty acid desaturase. Chem Pharm Bull. 2019;67(4):327-32.

29 Danso M, Boiten W, van Drongelen V, Gmelig Meijling K, Gooris G, El Ghalbzouri A, et al. Altered expression of epidermal lipid bio-synthesis enzymes in atopic dermatitis skin is accompanied by changes in stratum corneum lipid composition. J Dermatol Sci. 2017;88(1):57-66.

30 Dumas SN, Guo CA, Kim JK, Friedline RH, Ntambi JM. Interleukin-6 derived from cutaneous deficiency of stearoyl-CoA desaturase-1 may mediate metabolic organ crosstalk among skin, adipose tissue and liver. Biochem Biophys Res Commun. 2019;508(1):87-91.

31 Paton CM, Ntambi JM. Biochemical and physiological function of stearoyl-CoA desaturase. Am J Physiol Endocrinol Metab. 2009; 297(1):E28-37.

32 Enoch HG, Catalá A, Strittmatter P. Mechanism of rat liver microsomal stearyl-CoA desaturase. Studies of the substrate specificity, enzyme-substrate interactions, and the function of lipid. J Biol Chem. 1976;251(16):5095103.

33 Downing DT, Colton SW. Skin surface lipids of the horse. Lipids. 1980;15(5):323-7.

34 Frost ML, Colton SW, Wertz PW, Downing DT. Structures of the dienoic lactones of horse sebum. Comp Biochem Physiol B. 1984;78(3): 549-52.

35 Cameron DJ, Tong Z, Yang Z, Kaminoh J, Kamiyah $\mathrm{S}$, Chen $\mathrm{H}$, et al. Essential role of ELOVL4 in very long chain fatty acid synthesis, skin permeability barrier function, and neonatal survival. Int J Biol Sci. 2007;3(2): $111-9$.

36 Hopiavuori BR, Anderson RE, Agbaga M-P. ELOV4: very long-chain fatty acids serve an eclectic role in mammalian health and function. Prog Retinal Eye Res. 2019;69:137-58.
37 Ohno Y, Nakamichi S, Ohkuni A, Kamiyama $\mathrm{N}$, Naoe A, Tsujimura H, et al. Essential role of the cytochrome P450 CYP4F22 in the production of acylceramide, the key lipid for skin permeability barrier formation. Proc Natl Acad Sci U S A. 2015;112(25):7707-12.

38 Hall AM, Wiczer BM, Herrmann T, Stremmel W, Bernlohr DA. Enzymatic properties of purified murine fatty acid transport protein 4 and analysis of acyl-CoA synthetase activities in tissues from FATP4 null mice. J Biol Chem. 2005;280(12):11948-54.

39 Ohno Y. Elucidation of the synthetic mechanism of acylceramide, an essential lipid for skin barrier function. Yakugaku Zasshi. 2017; 137(10):1201-8.

40 Yamamoto H, Hattori M, Chamulitrat W, Ohno Y, Kihara A. Skin permeability barrier formation by the ichthyosis-causative gene FATP4 through formation of the barrier lipid $\omega$-O-acylceramide. Proc Natl Acad Sci U S A. 2020;117(6):2914-22.

41 Radin NS. Biosynthesis of the sphingoid bases: a provocation. J Lipid Res. 1984;25(13): 1536-40.

42 Mizutani Y, Mitsutake S, Tsuji K, Kihara A, Igarashi Y. Ceramide biosynthesis in keratinocyte and its role in skin function. Biochimie. 2009;91(6):784-90.

43 Levy M, Futerman AH. Mammalian ceramide synthases. IUBMB Life. 2010;62(5): 347-56.

44 Jennemann R, Rabionet M, Gorgas K, Epstein S, Dalpke A, Rothermel U, et al. Loss of ceramide synthase 3 causes lethal skin barrier disruption. Hum Mol Genet. 2012;21(3):586608.

45 Robson KJ, Stewart ME, Michelsen S, Lazo ND, Downing DT. 6-Hydroxy-4-sphingenine in human epidermal ceramides. J Lipid Res. 1994;35(11):2060-8.

46 Madison KC, Sando GN, Howard EJ, True CA, Gilbert D, Swartzendruber DC, et al. Lamellar granule biogenesis: a role for ceramide glucosyltransferase, lysosomal enzyme transport, and the Golgi. J Investig Dermatol Symp Proc. 1998:3(2):80-6.

47 Ohno Y, Kamiyama N, Nakamichi S, Kihara A. PNPLA1 is a transacylase essential for the generation of the skin barrier lipid $\omega$-Oacylceramide. Nat Commun. 2017;8:14610.

48 Hirabayashi T, Anjo T, Kaneko A, Senoo Y, Shibata A, Takama $\mathrm{H}$, et al. PNPLA1 has a crucial role in skin barrier function by directing acylceramide biosynthesis. Nat Commun. 2017;8:14609.

49 Grond S, Eichmann TO, Dubrac S, Kolb D, Schmuth M, Fischer J, et al. PNPLA1 deficiency in mice and humans leads to a defect in the synthesis of omega-O-acylceramides. J Invest Dermatol. 2017;137(2):394-402.

50 Klein B, Grond S, Haemmerie G, Lass A, Eichmann TO, Radner FPW. ABHD5 stimulates PNPLA1-mediated $\omega$-O-acylceramide biosynthesis essential for a functional skin permeability barrier. J Lipid Res. 2018;59:23607. 
51 Wertz PW, Downing DT. Metabolism of linoleic acid in porcine epidermis. J Lipid Res. 1990;31(10):1839-44.

52 Flammersfeld A, Panyot A, Yamaryo-Botté Y, Aurass P, Przyborski JM, Flieger A, et al. A patatin-like phospholipase functions during gametocyte induction in the malaria parasite Plasmodium falciparum. Cell Microbiol. 2020;22(3):e13146.

53 Grayson S, Johnson-Winegar AG, Wintroub BU, Isseroff RR, Epstein EH, Elias PM. Lamellar body-enriched fractions from neonatal mice: preparative techniques and partial characterization. J Invest Dermatol. 1985; 85(4):289-94.

54 Wertz P. Epidermal lamellar granules. Skin Pharmacol Physiol. 2018;31(5):262-8.

55 Sakai K, Akiyama M, Sugiyama-Nakagiri Y, McMillan JR, Sawamura D, Shimizu H. Localization of ABCA12 from Golgi apparatus to lamellar granules in human upper epidermal keratinocytes. Exp Dermatol. 2007;16(11): 920-6.

56 Wertz PW, Downing DT, Freinkel RK, Traczyk TN. Sphingolipids of the stratum corneum and lamellar granules of fetal rat epidermis. J Invest Dermatol. 1984;83(3):193-5.

57 Raymond AA, Gonzalez de Peredo A, Stella A, Ishida-Yamamoto A, Bouyssie D, Serre G, et al. Lamellar bodies of human epidermis: proteomics characterization by high throughput mass spectrometry and possible involvement of CLIP-170 in their trafficking/secretion. Mol Cell Proteomics. 2008;7(11):215175.

58 Freinkel RK, Traczyk TN. Lipid composition and acid hydrolase content of lamellar granules of fetal rat epidermis. J Invest Dermatol. 1985;85(4):295-8.

59 Wertz PW. Epidermal lipids. Sem Dermatol. 1992;11:106-13.

60 Elias PM, Fartasch M, Crumrine D, Behne M, Uchida Y, Holleran WM. Origin of the corneocyte lipid envelope (CLE): observations in Harlequin ichthyosis and cultured human keratinocytes. J Invest Dermatol. 2000;115(4): 765-9.

61 Kelsell DP, Norgett EE, Unsworth H, Teh MT, Cullup T, Mein CA, et al. Mutations in ABCA12 underlie the severe congenital skin disease Harlequin ichthyosis. Am J Hum Genet. 2005;76(5):794-803.

62 Akayama M, Sugiyama-Nakagiri Y, Sakai K, McMillan JR, Goto M, Arita K, et al. Mutations in lipid transporter ABCA12 in Harlequin ichthyosis and functional recovery by corrective gene transfer. J Clin Invest. 2005; 115:1777-84.

63 Wertz PW, Madison KC, Downing DT. Covalently bound lipids of human stratum corneum. J Invest Dermatol. 1989;92(1):109-11.

64 Chernomordik LV, Kozlov MM. Mechanics of membrane fusion. Nat Struct Mol Biol. 2008;15(7):675-83.
65 Slater J, Hill JR, Wertz PW. Evidence indicating that the acylglucosylceramide in the bounding membrane of lamellar granules is oriented with the glucosyl moiety on the inside. J Dent Res. 2003;82A:760.

66 Landmann L, Wertz PW, Downing DT. Acylglucosylceramide causes flattening and stacking of liposomes. An analogy for assembly of the epidermal permeability barrier. Biochim Biophys Acta. 1984;778(3):412-8.

67 Abraham W, Wertz PW, Downing DT. Effect of epidermal acylglucosylceramides and acylceramides on the morphology of liposomes prepared from stratum corneum lipids. Biochim Biophys Acta. 1988;939(2):403-8.

68 Abraham W, Wertz PW, Downing DT. Fusion patterns of liposomes formed from stratum corneum lipids. J Invest Dermatol. 1988; 90(3):259-62.

69 Norlen L. Skin barrier formation: the membrane folding model. J Invest Dermatol. 2001; 117(4):823-9.

70 den Hollander L, Han H, de Winter M, Svensson L, Masich S, Daneholt B, et al. Skin lamellar bodies are not discrete vesicles but part of a tubuloreticular network. Acta Derm Venereol. 2016;96(3):303-8.

71 Norlen L. Skin barrier structure and function: the single gel phase model. J Invert Dermatol. 2001;117:830-6.

72 Narangifard A, den Hollander L, Wennberg CL, Lundborg M, Lindahl E, Iwai I, et al. Human skin barrier formation takes place via a cubic to lamellar lipid phase transition as analyzed by cryo-electron microscopy and EMsimulation. Exp Cell Res. 2018;366(2):13951.

73 Brash AR, Yu Z, Boeglin WE, Schneider C. The hepoxilin connection in the epidermis. FEBS J. 2007;274(14):3494-502.

74 Munoz-Garcia M, Thomas CP, Keeney DS, Zheng Y, Brash AR. The importance of the lipoxygenase-hepoxilin pathway in the mammalian epidermal barrier. Biochim Biophys Acta. 2014;1841:401-8.

75 Simard-Bisson C, Parent LA, Moulin VJ, Fruteau de Laclos B. Characterization of epidermal lipoxygenase expression in normal human skin and tissue-engineered skin substitutes. J Histochem Cytochem. 2018;66(11): 813-24.

76 Guneri D, Voegeli R, Munday MR, Lane ME, Rawlings AV. 12R-lipoxygenase activity is reduced in photodamaged facial stratum corneum: a novel activity assay indicates a key function in corneocyte maturation. Int J Cosmet Sci. 2019;41:274-80.

77 Yamanashi H, Boeglin WE, Morisseau C, Davis RW, Sulikowski GA, Hammock BD, et al. Catalytic activities of mammalian epoxide hydrolases with cis and trans fatty acid epoxides relevant to skin barrier function. J Lipid Res. 2018;59(4):684-95.
78 Chiba T, Thomas CP, Calcutt MW, Boeglin WE, O'Donnell VB, Brash AR. The precise structures and stereochemistry of trihydroxylinoleates and their significance in barrier function. Implications of an epoxide hydrolase in the transformations of linoleate. J Biol Chem. 2016;291:14540-54.

79 Nugteren DH, Christ-Hazelhof E, van der Beek A, Houtsmuller UM. Metabolism of linoleic acid and other essential fatty acids in the epidermis of the rat. Biochim Biophys Acta. 1985;834(3):429-36.

80 Nemes Z, Marekov LN, Fésüs L, Steinert PM. A novel function for transglutaminase 1: attachment of long-chain omega-hydroxyceramides to involucrin by ester bond formation. Proc Natl Acad Sci U S A. 1999;96(15):84027.

81 Takuya T, Hirabayashi T, Miyasaka Y, Kawamoto A, Okuno Y, Taguchi S, et al. SDR9C7 catalyzes critical dehydrogenation of acylceramides for skin barrier formation. J Clin Invest. 2020;130:890-903.

82 Potts RO, Francoeur ML. The influence of stratum corneum morphology on water permeability. J Invest Dermatol. 1991;96(4):4959.

83 Wertz PW, Madison KC, Downing DT. Covalently bound lipids of human stratum corneum. J Invest Dermatol. 1989;92(1):109-11.

84 Wertz PW, Downing DT. Stratum corneum: biological and biochemical considerations. Drugs Pharm Sci. 1989;35:1-22.

85 Madison KC, Swartzendruber DC, Wertz PW, Downing DT. Presence of intact intercellular lipid lamellae in the upper layers of the stratum corneum. J Invest Dermatol. 1987; 88(6):714-8.

86 Swartzendruber DC, Wertz PW, Kitko DJ, Madison KC, Downing DT. Molecular models of the intercellular lipid lamellae in mammalian stratum corneum. J Invest Dermatol. 1989;92(2):251-7.

87 White SH, Mirejovsky D, King GI. Structure of lamellar lipid domains and corneocyte envelopes of murine stratum corneum. An Xray diffraction study. Biochemistry. 1988; 27(10):3725-32.

88 Bouwstra JA, Gooris GS, van der Spek JA, Bras W. Structural investigations of human stratum corneum by small-angle X-ray scattering. J Invest Dermatol. 1991;97(6):100512.

89 Hill JR, Wertz PW. Molecular models of the intercellular lipid lamellae from epidermal stratum corneum. Biochim Biophys Acta. 2003;1616(2):121-6.

90 Wertz PW, Swartzendruber DC, Kitko DJ, Madison KC, Downing DT. The role of the corneocyte lipid envelopes in cohesion of the stratum corneum. J Invest Dermatol. 1989; 93(1):169-72.

91 Chapman SJ, Walsh A, Jackson SM, Friedmann PS. Lipids, proteins and corneocyte adhesion. Arch Dermatol Res. 1991;283(3):16773. 
92 Crumrine D, Khnykin D, Krieg P, Man MQ, Celli A, Mauro TM, et al. Mutations in recessive congenital ichthyoses illuminate the origin and functions of the corneocyte lipid envelope. J Invest Dermatol. 2019;139(4): 760-8.

93 Chang F, Swartzendruber DC, Wertz PW, Squier CA. Covalently bound lipids in keratinizing epithelia. Biochim Biophys Acta. 1993;1150(1):98-102.

94 Wertz PW, Downing DT. Ceramidase activity in porcine epidermis. FEBS Lett. 1990; 268(1):110-2.

95 Yada Y, Higuchi K, Imokawa G. Purification and biochemical characterization of membrane-bound epidermal ceramidases from guinea pig skin. J Biol Chem. 1995;270(21): 12677-84

96 Houben E, Holleran WM, Yaginuma T, Mao C, Obeid LM, Rogiers V, et al. Differentiation-associated expression of ceramidase isoforms in cultured keratinocytes and epidermis. J Lipid Res. 2006;47(5):1063-70.

97 Wertz PW, Downing DT. Free sphingosines in porcine epidermis. Biochim Biophys Acta. 1998;1002(2):213-7.

98 Wertz PW, Downing DT. Free sphingosine in human epidermis. J Invest Dermatol. 1990;94(2):159-61.

99 Stewart ME, Downing DT. Free sphingosines of human skin include 6-hydroxysphingosine and unusually long-chain dihydrosphingosines. J Invest Dermatol. 1995; 105(4):613-8.

100 Arikawa J, Ishibashi M, Kawashima M, Takagi Y, Ichikawa Y, Imokawa G. Decreased levels of sphingosine, a natural antimicrobial agent, may be associated with vulnerability of the stratum corneum from patients with atopic dermatitis to colonization by Staphylococcus aureus. J Invest Dermatol. 2002;119(2):433-9.

101 Fischer CL, Wertz PW. Effects of endogenous lipids on the skin microbiome. In: Day- an N, editor. The skin microbiome: from basic research to product development. Hoboken, NJ: Wiley; 2020. p. 219-35.

102 Fischer CL, Drake DR, Dawson DV, Blanchette DR, Brogden KA, Wertz PW. Antibacterial activity of sphingoid bases and fatty acids against Gram-positive and Gram-negative bacteria. Antimicrob Agents Chemother. 2012;56(3):1157-61.

103 Bibel DJ, Aly R, Shah S, Shinefield HR Sphingosines: antimicrobial barriers of the skin. Acta Derm Venereol. 1993;73(6):40711.

104 Hannun YA, Loomis CR, Merrill AH, Bell RM. Sphingosine inhibition of protein kinase $\mathrm{C}$ activity and of phorbol dibutyrate binding in vitro and in human platelets. $J$ Biol Chem. 1986;261(27):12604-9.

105 Steen Law SL, Squier CA, Wertz PW. Free sphingosine in oral epithelium. Comp Biochem Physiol. 1995;110B:511-3.

106 Hennings H, Elgjo K. Epidermal regeneration after cellophane tape stripping of hairless mouse skin. Cell Tissue Kinet. 1970;3(3): 243-52.

107 Melton JL, Wertz PW, Swartzendruber DC, Downing DT. Effects of essential fatty acid deficiency on epidermal $\mathrm{O}$-acylsphingolipids and transepidermal water loss in young pigs. Biochim Biophys Acta. 1987;921(2): 191-7.

108 Weerheim A, Ponec M. Determination of stratum corneum lipid profile by tape stripping in combination with high-performance thin-layer chromatography. Arch Dermatol Res. 2001;293(4):191-9.

109 Radner FP, Marrakchi S, Kirchmeier P, Kim GJ, Ribierre F, Kamoun B, et al. Mutations in CERS3 cause autosomal recessive congenital ichthyosis in humans. PLoS Genet. 2013;9(6):e1003536.

110 Mueller N, Sassa T, Morales-Gonzalez S, Schneider J, Salchow DJ, Seelow D, et al. De novo mutation in ELOVL1 causes ichthyo- sis, acanthosis nigricans, hypomyelination, spastic paraplegia, high frequency deafness and optic atrophy. J Med Genet. 2019;56(3): 164-75.

111 Kutkowska-Kazmierczak A, Rydzanicz M Chlebowski A, Klosowska-Kosicka K, Mika A, Gruchota J, et al. Dominant ELOV1 mutation causes neurological disorder with ichthyotic keratoderma, spasticity, hypomyelination and dysmorphic features. J Med Genet. 2018;55:408-14.

112 Aldahmesh MA, Mohamed JY, Alkuraya HS, Verma IC, Puri RD, Alaiya AA, et al. Recessive mutations in ELOVL4 cause ichthyosis, intellectual disability, and spastic quadriplegia. Am J Hum Genet. 2011;89(6):74550.

113 Agbaga MP. Different mutations in ELOVL4 affect very long chain fatty acid biosynthesis to cause variable neurological disorders in humans. Adv Exp Med Biol. 2016;854:12935 .

114 Agbaga MP, Brush RS, Mandal MN, Henry $\mathrm{K}$, Elliott MH, Anderson RE. Role of Stargardt-3 macular dystrophy protein (ELOVL4) in the biosynthesis of very long chain fatty acids. Proc Natl Acad Sci U S A. 2008; 105(35):12843-8.

115 Jobard F, Lefevre C, Karaduman A, Blanchet-Bardon C, Emre S, Weissenbach J, et al Lipoxygenase-3 (ALOXE3) and 12(R)-lipoxygenase (ALOX 12B) are mutated in non-bullous ichthyosiform erythroderma (NCIE) linked to chromosome 17p13.1. Hum Mol Genet. 2002;11:107-13.

116 Russel LJ, DiGiovanna JJ, Rogers GR, Steinert PM, Hashem N, Compton JG, et al. Mutations in the gene for transglutaminase 1 in autosomal recessive lamellar ichthyosis. Nat Genet. 1995;9:279-83.

117 Huber M, Rettler I, Bernasconi K, Frenk E, Lavrijsen SP, Ponec M, et al. Mutations of keratinocyte transglutaminase in lamellar ichthyosis. Science. 1995;267(5197):525-8. 